\title{
NEW PROPERTIES AND REPRESENTATIONS FOR MEMBERS OF THE POWER-VARIANCE FAMILY. I
}

\author{
Vladimir Vinogradov*, Richard B. Paris ${ }^{\dagger}$ And Olga Yanushkevichiene ${ }^{\ddagger}$
}

\begin{abstract}
We derive new Wright-function representations for the densities of the generating measures of most representatives of the power-variance family of distributions. For all members of this family, we construct new saddlepoint-type approximations having an arbitrary fixed number of refining terms. To this end, we derive new, "exponentially small" Poincaré series for a subclass of the Wright functions, whose coefficients are expressed in terms of the Zolotarev polynomials.
\end{abstract}

Key Words: Difference quotient; Poincaré series; Poisson-gamma laws; reciprocity; refined saddlepoint approximations; stable laws; Stokes phenomenon; Wright function; Zolotarev duality.

MSC primary 60E07, 60E10, 60F10, 62E10, 62E20; secondary 33C10, 33C15, 33E20, 41A60

\section{Introduction}

This is the first part of a series of two articles. The second part quoted as reference [23] is referred to as [VPY2] throughout this article. Throughout this paper, we employ the notation and terminology developed in Vinogradov (2004a) whenever possible.

Tweedie (1984) introduced an important class of exponential dispersion models (or EDM's) which now bears his name. In that paper, he also discussed informally their relationships with the Wright function as well as with Zolotarev's early work on stable distributions. See Tweedie (1984, Sec. 5) for more detail. Since then, numerous connections between those Tweedie EDM's which correspond to the range of values of the power parameter $p \in(-\infty, 0] \cup(2,+\infty)$ and the extreme stable distributions with the index of stability $\alpha \in(0,1) \cup(1,2]$ have been established (cf., for example, Jørgensen (1997, Subsec. 4.2.2) or Vinogradov (2004a)). In contrast, a relationship between Tweedie EDM's and the Wright function has not been adequately addressed yet. Therefore, one of our goals is to fill in this gap (see Section 3). A generic EDM comprised of specific infinitely divisible distributions can be regarded as the class of all marginals of the exponential family of certain univariate Lévy processes. See Hochberg and Vinogradov (2009, p. 251) for more detail. The Wright function is introduced in Definition 2.1.

The totality of all the members of the Tweedie EDM's comprises the three-parameter powervariance family (or the PVF) of the univariate probability distributions. This choice of an alternative name for the Tweedie family is justified by the variance-to-mean relationship (3.5), which has the power type. This relationship involves the so-called power parameter $p$ whose domain is

$$
\Delta:=\mathbf{R}^{1} \backslash(0,1)
$$

\footnotetext{
*Department of Mathematics, Ohio University, Athens, OH, 45701 USA, e-mail: vinograd@ohio.edu

${ }^{\dagger}$ School of Computing, Engineering and Applied Mathematics, University of Abertay Dundee, Dundee, DD1 1HG, UK, e-mail: r.paris@abertay.ac.uk

${ }^{\ddagger}$ Institute of Mathematics and Informatics, Vilnius University, Akademijos str., 4, LT-08663 Vilnius, Lithuania, e-mail: olga.januskeviciene@mii.vu.lt
} 
see Jørgensen (1997, form. (4.1)-(4.2)), Vinogradov (2004a, form. (1.6)), or (2.4).

Section 2 deals with the review of relevant auxiliary results. Section 3 contains new representations for Tweedie EDM's as well as several new concepts and technical assertions. Some of them are of independent interest. For instance, we propose a new approach, which involves the concept of the difference quotient, for studying the properties of the scaled Poisson EDM, which corresponds to $p=1$ (see Definition 3.1 and Remark 3.2). The other noticeable results of that section are Proposition 3.6 and formulas (3.14) and (3.25).

A distinct feature of this paper is its reliance on analytical methods and in particular, the machinery of special functions, for tackling the problems of Probability Theory. Such synthesis of the ideas and methods of Probability Theory and the Theory of Special Functions had already proved to be effective for providing a simple derivation of new assertions of Probability Theory, as well as for finding a meaningful interpretation of particular analytical results of the Theory of Special Functions (cf., for example, Vinogradov (2007a, Sec. 3)). A similar approach is also pursued here. Such connections frequently are a two-way interaction. Thus, some of our new Probability Theory results yield previously unknown assertions of the Theory of Special Functions (cf., for example, (3.19)). This is illustrated further in [VPY2, Sec. 4] where three standard special functions are employed.

The proof of some of our new results of Probability Theory required the derivation of a previously unknown subtle property on the asymptotic behavior of a specific member of the class of general Wright functions, which is introduced in [VPY2, Def. 2.5]. This assertion is given as [VPY2, form. (5.8)]. Since its proof is too long, it is dealt with separately in Paris (2011b).

In this paper, we had no choice but to include numerous modifications of the results which were previously known but presented in a less customary context. Naturally, this is the price we had to pay for merging the techniques of two branches of Mathematics i.e., Probability Theory and the Theory of Special Functions, which for a long time have been developing along separate paths. The other inconvenience caused by this synthesis of the methods of different branches of Mathematics is the necessity to employ as many as three closely related parameters, namely, $p, \alpha$ and $\rho$ instead of only one (The latter two parameters are defined by (2.6)-(2.7).)

Numerous subtle properties of the stable laws were derived previously by employing elaborate analytical methods, but with no reference to the Wright function. Apparently, Ibragimov, Linnik and Zolotarev who established important properties of the extreme stable laws, overlook their connection with the Wright function. We trust that our persistence in revealing the links between the PVF and the Wright function will help understand some properties of the Tweedie class on a deeper level. In addition, we demonstrate that various distribution theory results derived by these scholars are equivalent to previously unknown representations in the Theory of Special Functions. Hence, experts in Analysis no longer regard distribution theory as a peripheral area of Analysis. Indeed, this "branch" is quite capable of providing an impact on other domains of Analysis.

Remark 3.3 discusses how the members of the subclass of (2.2) of the Wright functions characterized by $\rho \in(-1,0)$ may be used to obtain the closed-form expression for the probability density functions (or p.d.f.'s) of both the positive stable distributions with the index of stability $\alpha=-\rho \in(0,1)$ and the skewness parameter $\beta=1$, and the extreme stable distributions with $\alpha \in(1,2)$ and $\beta=-1$. In turn, the expressions of such character can be utilized for the derivation of certain representations for the Wright function in terms of standard special functions. This approach enabled us to establish the identities [VPY2, form. (4.1), (4.9) and (4.16)] starting from the previously available representations for the positive stable and Poisson-exponential distributions. Note that although the analytical proof of these three identities (which formally are the results of the Theory of Special Functions) is straightforward, they were previously unnoticed.

The main result of the first part of this paper is Theorem 4.8. It provides the refined saddlepoint- 
type approximations for all members of the Tweedie EDM's. We demonstrate that each such approximation may contain an arbitrary fixed number of refining terms of decreasing magnitude, discuss the recursion for their generation as well as provide the first five terms. Theorem 4.8 pertains to the absolutely continuous, purely discrete and mixed cases.

In order to explain the meaning of Theorem 4.8 and a related conjecture, we now review the concept of the infinite asymptotic series and the relevant notation, which are due to Poincaré (1886). In what follows, the expression of the type " $f(z) \sim \sum_{k=0}^{\infty} \ldots$ " is understood as that which provides the Poincaré asymptotic series for a function $f(z)$ at a specified point. We refer to Olver (1997, pp. 16-17) or Paris (2011a) for a comprehensive discussion and numerous examples on Poincaré series, and to Ibragimov and Linnik (1971, p. 55) or Zolotarev (1986, Th. 2.5.2) for some examples on the use of such infinite expansions in Probability Theory.

Now, we give a deeper insight into Theorem 4.8. Its main novelty pertains to the case where $p \in(1,2)$ (or $\rho>0$ ), which corresponds to the Poisson-gamma subclass of the family of Tweedie EDM's. The members of this subclass are characterized by (3.23)-(3.25). In this case, Theorem 4.8.ii complements the algorithms developed by Dunn and Smyth (2005, 2008) for computing the densities of the absolutely continuous components of the Poisson-gamma distributions. A comparison between our approximations and those proposed by Dunn and Smyth $(2005,2008)$ is addressed in Remark 4.11. The evaluation of the Poisson-gamma "densities", which can be done by using Theorem 4.8.ii2 and formulas (4.17) and (4.20) is an important task for the Property and Casualty Insurance, since the totality of the class of the Poisson-gamma probability laws is widely regarded as the most appropriate model for the accumulated claims. For this reason, Theorem 4.8.ii2 and Corollary 4.9.ii, which can be interpreted as the results on large deviations (or excessively large accumulated claims), might have potential applications in reinsurance.

In the case where $p \in\{1 ; 2\}$, the refining terms which emerge in (4.16) and (4.18) are expressed by virtue of the Stirling coefficients, i.e., the coefficients of the Poincaré series for the reciprocal of the gamma function. We refer to Paris and Kaminski (2001, form. (2.1.11)-(2.1.12)) for their definition and recursive evaluation. See also (4.8)-(4.10).

In the case where $p \in \Delta \backslash\{1 ; 2\}$, the refining terms which appear in (4.17) are expressed by means of a transformation of the polynomials which we call the Zolotarev polynomials. These polynomials are defined recursively by (4.12). However, we were able to find them in closed form in three special cases (see [VPY2, form. (4.7), (4.15), (4.21]). Remark 4.10 contains a conjecture on our refined saddlepoint-type approximations, which is equivalent to a relationship between Zolotarev polynomials and the Stirling coefficients. We use Mathematica for the recursive evaluation of the specific coefficients of the asymptotic expansions and the numerical verification of the plausibility of this conjecture.

In the case where $p \in(-\infty, 0] \cup(2,+\infty)$, the proof of Theorem 4.8 relies on an application of the results by Zolotarev (1986, Sec. 2.5); see also Ibragimov and Linnik (1971, Sec. 2.4). We warn that some of these just quoted results contain misprints (compare Zolotarev (1986, p. 257, comments 2.12-2.13)). Incidentally, we also discovered and corrected several minor misprints contained in Zolotarev (1986); see Remarks 3.4 and 4.3.i. Throughout Section 4, we discuss the interplay of our results with those presented in Ibragimov and Linnik (1971, Sec. 2.4), and Zolotarev (1986, Sec. 2.5).

For $p \in(1,2)$, the proof of Theorem 4.8.ii involves Lemma 4.6, which pertains to the subclass (2.2) of the Wright functions $\phi(\rho, 0, z)$. It stipulates that the refining terms of the "exponentially small" Poincaré asymptotic series for the functions $\{\phi(\rho, 0, z), \rho \in(-1,0)\}$ as $z \rightarrow-\infty$, and $\{\phi(\rho, 0, z), \rho>0\}$ as $z \rightarrow+\infty$, can be written down in a similar form (see (4.14)-(4.15)). Remark 4.7 discusses a relationship between Lemma 4.6.i and Withers and Nadarajah (2011). That lemma is a new result in the Theory of Special Functions, where it might have a particular value. The 
proof of Lemma 4.6.i is deferred to Appendix A (see [VPY2, Sec. 5]). The concluding Appendix B contains the proof of Theorem 4.8 (see [VPY2, Sec. 6]).

Apart from the refined saddlepoint-type approximations constructed in Section 4, the other main feature of this work pertains to finding an interpretation for the Letac-Mora reciprocity for certain pairs of the natural exponential families (or NEF's), which are comprised of the specific members of Tweedie EDM's. See (4.2)-(4.4) for a description of the Letac-Mora reciprocity. Moreover, the main result of the second part of this work, namely, [VPY2, Th. 3.1] connects this concept with subtle properties of the Poincaré series constructed in Theorem 4.8.

In [VPY2, Sec. 4], we consider the three illustrative examples to Theorem 4.8.ii2-ii3, which are related to particular Bessel and Whittaker functions. In the same section, we give four representations in terms of standard special functions for the densities of the Tweedie EDM's which correspond to $p \in\{-1 ; 3 / 2 ; 5 / 2 ; 4\}$ (see [VPY2, form. (4.8), (4.17), (4.13), and (4.5)], respectively).

In order to reduce the length of this paper, we omitted the description of those concepts that are routine for the theory of EDM's and NEF's. However, in order to facilitate the reading, we now provide the references to more comprehensive work pertaining to specific topics discussed. In particular, we refer to Jørgensen (1997) for the consideration of EDM's and NEF's, and to Zolotarev (1986) or Uchaikin and Zolotarev (1999) for univariate stable distributions. In the non-negative case, the concepts of EDM's and NEF's are reviewed and illustrated in the Probability Theory setting by Hochberg and Vinogradov (2009, Def.'s 3-5). The proofs of some results are rather routine involving a substantial amount of Calculus and very basic Probability Theory. In such cases, we have left technicalities to the reader presenting only the main ideas of the proofs.

\section{Auxiliary definitions}

Here, we assemble those auxiliary concepts and results which will be employed in the sequel.

First, log stands for the natural logarithm of the real or complex argument (In the complex case, $\log z$ is understood as its principal value). An empty sum or product is interpreted as zero or unity, respectively, whereas $a \vee b:=\max (a, b)$. Also, $\mathbf{Z}_{+}, \mathbf{N}$, and $\mathbf{R}_{+}^{1}$ stand for the sets of all non-negative and all positive integers, and all positive reals, respectively.

Definition 2.1 The complex-valued Wright function $\phi(\rho, \delta, z)$ of argument $z \in \mathbb{C}$, which is indexed by the real-valued parameter $\rho \in(-1,0) \cup(0, \infty)$ and the complex-valued parameter $\delta \in \mathbb{C}$ is hereinafter defined by the following convergent series:

$$
\phi(\rho, \delta, z):=\sum_{k=0}^{\infty} \frac{z^{k}}{k ! \cdot \Gamma(\rho \cdot k+\delta)} .
$$

We refer to Braaksma (1963), Gorenflo et al. (1999), and Paris and Kaminski (2001, Sec. 2.3) for a comprehensive treatment of the properties of this function.

The Tweedie EDM's are related only to those members of class (2.1) for which $\delta=0$. This point of view is developed further in Section 3. Under this assumption, it follows from the fact that the gamma function has a pole at the origin that the first term of the infinite series which emerges on the right-hand side of (2.1) can be dropped, that is,

$$
\phi(\rho, 0, z) \equiv \sum_{k=1}^{\infty} \frac{z^{k}}{k ! \cdot \Gamma(\rho \cdot k)} .
$$

Therefore, we will limit our consideration to the subclass (2.2) only. Moreover, everywhere except the proof of Lemma 4.6 we assume that the argument $z$ of $\phi(\rho, 0, z)$ takes on real values. It is clear 
that for values of $\rho>0,(2.2)$ yields that as $z \rightarrow 0$,

$$
\phi(\rho, 0, z)=\sum_{k=1}^{[1 / \rho] \vee 1} \frac{z^{k}}{k ! \cdot \Gamma(\rho \cdot k)}+\mathcal{O}\left(|z|^{[1 / \rho] \vee 1+1}\right) .
$$

The proof of Lemma 4.6 involves the use of the subtle asymptotic properties of a more complicated (as compared to (2.1)) general Wright function, which is introduced in [VPY2, form. (2.4)]. Relevant properties of that function are established in Appendix A.

Next, we discuss some aspects of the PVF, which is hereinafter denoted by

$$
\left\{T w_{p}(\mu, \lambda)\right\}\left(=\left\{T w_{p}(\mu, \lambda), p \in \Delta, \mu \in \Omega_{p}, \lambda \in \mathbf{R}_{+}^{1}\right\}\right) .
$$

By Jørgensen (1997, Prop. 4.2), the Tweedie EDM's which correspond to $p \in(0,1)$ do not exist. In what follows, $\mu$ and $\lambda$ stand for the mean (or location) and scaling parameters, respectively. Each member $T w_{p}(\mu, \lambda)$ of the PVF is characterized by arbitrary fixed admissible values of $p, \mu$ and $\lambda$. A rigorous description of this family, including the domain $\Omega_{p}$ of the location parameter, is deferred to Section 3. At this point, let us just mention that it follows from Jørgensen (1997, Th. 4.1) that given $p \in \Delta$, the two-parameter family $\left\{T w_{p}(\mu, \lambda), \mu \in \Omega_{p}, \lambda \in \mathbf{R}_{+}^{1}\right\}$ comprises a reproductive EDM with the following unit-variance function (or u.v.f.):

$$
\mathbf{V}_{p}(\mu)=\mu^{p}
$$

In view of the synthesis of the methods used in the different branches of Mathematics which is pursued in this paper, we have to employ the following two parameters, which are closely related to $p$. Thus, the parameter $\rho$ of the function $\phi(\rho, 0, z)$, whose domain is the set $(-1,0) \cup(0, \infty)$, and the power parameter $p$ (which characterizes a particular model within the class of all Tweedie EDM's) are in one-to-one correspondence described by the following formula:

$$
\rho\left(=\rho_{p}\right):=(2-p) /(p-1) .
$$

The function $\rho_{p}$ given by (2.6) maps $\Delta \backslash\{1\}$ onto $(-2,+\infty) \backslash\{-1\}$. However, this parameter corresponds to a specific Wright function from subclass $(2.2)$ only for $p \in(-1,+\infty) \backslash\{0\}$.

It is sometimes more convenient to use the index of stability $\alpha=-\rho$ instead of $\rho$. Therefore, given $p \in \Delta \backslash\{1\}$, we introduce the parameter

$$
\alpha\left(=\alpha_{p}\right):=-\rho_{p}=(p-2) /(p-1) .
$$

It follows by reflection that the function $\alpha_{p}$ defined by (2.7) maps $\Delta \backslash\{1\}$ onto $(-\infty, 2) \backslash\{1\}$. But this parameter corresponds to a stable law with index $\alpha \in(0,1) \cup(1,2]$ only for $p \in(-\infty, 0] \cup(2,+\infty)$. Hence, the use of $\rho_{p}$ is justified for the range of $p \in(1,2) \cup(2,+\infty)$, whereas it makes sense to employ $\alpha_{p}$ in the range of $p \in(-\infty, 0] \cup(2,+\infty)$ only. In what follows, we will utilize both $\alpha_{p}$ and $\rho_{p}$, since the former parameter is convenient for describing the relations of the PVF with the extreme stable laws, whereas the latter should be employed for characterizing the connections between Tweedie EDM's and the Wright function.

Next, we discuss the concepts relevant to the unit deviance, which is defined by (2.10) (see also Jørgensen (1997, Def. 1.1, Subsec.'s 3.1.3 and 4.1.3), and Dunn and Smyth (2008, form. (3)) for more detail). Set

$$
H_{p}(y):= \begin{cases}(0 \vee y)^{2-p} /((1-p) \cdot(2-p)) & \text { if } p \in \Delta \backslash\{0 ; 1 ; 2\} \\ y^{2} / 2 & \text { if } p=0 \\ y \cdot \log y & \text { if } p=1 \\ -\log y & \text { if } p=2 .\end{cases}
$$


The domain of this function is $\mathbf{R}_{+}^{1}$ if $p \in\{1 ; 2\}$. For the remaining values of $p \in \Delta \backslash\{1 ; 2\}, y \in \mathbf{R}^{1}$. It follows from (2.8) that for $y \in \mathbf{R}_{+}^{1}, H_{p}^{\prime \prime}(y)=y^{-p}$. Also, define

$$
\begin{gathered}
\mathcal{E}_{p}(y, \mu):=H_{p}(y)-H_{p}(\mu)-H_{p}^{\prime}(\mu) \cdot(y-\mu) \\
= \begin{cases}\frac{(0 \vee y)^{2-p}}{(1-p)(2-p)}-\frac{\mu^{1-p} y}{(1-p)(2-p)}+\frac{\mu^{2-p}}{2-p} & \text { if } p \in \Delta \backslash\{0 ; 1 ; 2\} ; \\
(y-\mu)^{2} / 2 & \text { if } p=0 ; \\
y \cdot \log (y / \mu)-(y-\mu) & \text { if } p=1 ; \\
-\log (y / \mu)+(y-\mu) / \mu & \text { if } p=2 .\end{cases}
\end{gathered}
$$

For each fixed $p \in \Delta$, the function $\mathcal{E}_{p}(y, \mu)$ is hereinafter referred to as the Weierstrass function which is constructed starting from the function $H_{p}(y)$. We refer to Ioffe and Tihomirov (1979, Subsec. 2.2.3) for more detail on the properties of this function and in particular, p. 114, form. (15) therein. It is easily seen that in the "border", extreme stable cases, which are characterized by the values of $\{p<0 ; \mu=0\}$ and $\{p>2 ; \mu=+\infty\}$, the second and the third terms which emerge in the top formula on the right-hand side of (2.9) are to be equated to 0 .

Given $p \in \Delta$, we emphasize the following relationship between the Weierstrass function $\mathcal{E}_{p}(y, \mu)$ and the unit deviance $d_{p}(y, \mu)$, which corresponds to the $\operatorname{EDM}\left\{T w_{p}(\mu, \lambda), \mu \in \Omega_{p}, \lambda \in \mathbf{R}_{+}^{1}\right\}$ :

$$
d_{p}(y, \mu) \equiv 2 \cdot \mathcal{E}_{p}(y, \mu) .
$$

The Weierstrass function (2.9) will be used to stress the exponential decay of the function $f_{p, \mu, \lambda}(y)$, which has the magnitude $\lambda \cdot \mathcal{E}_{p}(y, \mu)$ (see (4.1) and (4.17)-(4.18)).

To conclude this section, we summarize some useful notation and terminology. Thus, we often follow the custom of formulating various statements of distribution theory in terms of the properties of r.v.'s, even when such results pertain only to their distributions. The acronyms "ch.f." and "c.g.f." are used for characteristic and a cumulant-generating functions, respectively. In what

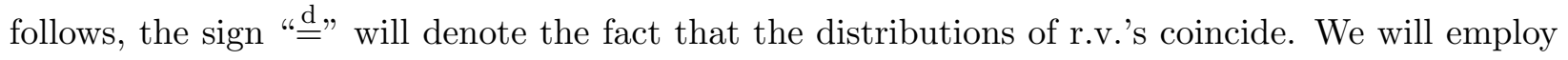
the Pochhammer symbol $(a)_{j}$, which is defined for positive integer $j$ by

$$
(a)_{0}:=1, \quad(a)_{j}:=\Gamma(a+j) / \Gamma(a)=a \cdot(a+1) \cdot(a+2) \cdot \ldots \cdot(a+j-1) .
$$

\section{Tweedie EDM's and their Wright-Function Representations}

The description of the Tweedie EDM's and the PVF in terms of the c.g.f.'s of its members can be found in Vinogradov (2004a, Prop. 1.1). Here, an emphasis is made on the description of the PVF by virtue of the distributional properties of the probability laws which comprise this family, since it is more convenient for our needs. An exception is formula (3.36), which easily follows from Vinogradov (2004a, Prop. 1.1.iii).

A related approach is to characterize the generating measure of each individual Tweedie EDM. We refer to Jørgensen (1997, p. 71) or Jørgensen et al. (2009, Subsec. 4.1) for the definition of the generating measure in the general case. To some extent, a similar line of development was pursued in a different context of describing the domains of attraction to Tweedie laws by Jørgensen et al. (2009, Sec. 5). For a fixed $p \in(-\infty, 0] \cup(2, \infty)$, the generating measure of the Tweedie EDM $\left\{T w_{p}(\mu, \lambda), \mu \in \Omega_{p}, \lambda \in \mathbf{R}_{+}^{1}\right\}$ is characterized by its density. This density is equal to the p.d.f. $f_{p, 0,1}(y)$ (if $\left.p \in(-\infty, 0]\right)$ and to the p.d.f. $f_{p, \infty, 1}(y)$ (if $p \in(2, \infty)$ ), which are defined by $(3.11)$ and (3.10), respectively.

For each $p \in(1,2)$, the topological support of the generating measure of the Tweedie EDM $\left\{T w_{p}(\mu, \lambda), \mu \in \Omega_{p}, \lambda \in \mathbf{R}_{+}^{1}\right\}$ is $[0,+\infty)$. The structure of this measure is such that it has point mass 
1 at the origin and the density over $\mathbf{R}_{+}^{1}$, for which we discovered a new closed-form representation. Namely, the density of this generating measure is expressed in terms of the Wright function as follows: $y^{-1} \cdot \phi\left(\rho_{p}, 0, B_{p, 1} \cdot y^{\rho_{p}}\right)$ (compare $\left.(3.25)\right)$.

In the sequel, the scaling parameter $\lambda \in \mathbf{R}_{+}^{1}$. The domain $\Omega_{p}$ of the location parameter is such that $\Omega_{p}=[0, \infty)$ if $p \in(-\infty, 0) ; \Omega_{0}=\mathbf{R}^{1} ; \Omega_{p}=\mathbf{R}_{+}^{1}$ for $p \in[1,2]$, and $\Omega_{p}=(0, \infty]$ if $p \in(2, \infty)$. In what follows, we will refer to the values of the parameters specified above as their admissible values.

The PVF includes normal distributions $(p=0)$, gamma distributions $(p=2)$, inverse Gaussian distributions $(p=3)$, and non-central gamma distributions with zero degrees of freedom $(p=3 / 2)$. The scaled Poisson distributions correspond to $p=1$ (see (3.1)). In turn, Tweedie distributions with $p \in(2, \infty)$ are derived by an exponential tilting of positive stable laws with the index of stability $\alpha_{p} \in(0,1)$ given by $(2.7)$. The members of the PVF which correspond to $p \in(1,2)$ are characterized by the compound Poisson representation (3.23).

The simplest classes of the scaled Poisson and the gamma distributions are reviewed first.

It is known that for arbitrary fixed $\mu \in \mathbf{R}_{+}^{1}$ and $\lambda \in \mathbf{R}_{+}^{1}$,

$$
T w_{1}(\mu, \lambda) \stackrel{\mathrm{d}}{=} \lambda^{-1} \cdot T w_{1}(\mu \cdot \lambda, 1) \stackrel{\mathrm{d}}{=} \lambda^{-1} \cdot \operatorname{Poiss}\left(\Phi_{1}\right),
$$

where the parameter $\Phi_{1}:=\lambda \cdot \mu$ (compare to (3.6) and Vinogradov (2004a, form. (2.9)).

Definition 3.1 Given $\mu \in \mathbf{R}_{+}^{1}, \lambda \in \mathbf{R}_{+}^{1}$ and $y \in[0, \infty)$, we introduce the following quantity and hereinafter term it the difference quotient:

$$
f_{1, \mu, \lambda}(y):= \begin{cases}\lambda \cdot \mathbf{P}\left\{T w_{1}(\mu, \lambda)=y\right\}=\lambda \cdot e^{-\Phi_{1}} \cdot \Phi_{1}^{y \cdot \lambda} /(y \cdot \lambda) ! & \text { if } y \in \lambda^{-1} \cdot \mathbf{Z}_{+} \\ 0 & \text { otherwise }\end{cases}
$$

Remark 3.2 The rationale for calling expression (3.2) the "difference quotient" is due to the fact that in the most important case where $y \in \lambda^{-1} \cdot \mathbf{Z}_{+}$, one obtains that

$$
f_{1, \mu, \lambda}(y)=\mathbf{P}\left\{y-1 / \lambda<T w_{1}(\mu, \lambda) \leq y\right\} /(1 / \lambda) .
$$

Proposition 3.6 contains a scaling property of this quotient. See also (3.30)-(3.33), (4.16), (4.19) and [VPY2, Th. 3.1.ii] for the other relevant relationships. Some of them stipulate that although the purely discrete scaled Poisson distribution is spanned over the lattice $\lambda^{-1} \cdot \mathbf{Z}_{+}$, the transformation (3.3) spreads the positive mass located at a point on this lattice uniformly over an interval of length $1 / \lambda$. The consideration of $f_{1, \mu, \lambda}(y)$ helps reveal certain new asymptotic properties of this class of discrete distributions as $\lambda \rightarrow \infty$, which happen to be closely related to those of the other representatives of the PVF which possess an absolutely continuous component.

Next, the value of $p=2$ ( or $\rho=0$ ) corresponds to the gamma EDM, which is hereinafter denoted by $\left\{T w_{2}(\mu, \lambda), \mu \in \mathbf{R}_{+}^{1}, \lambda \in \mathbf{R}_{+}^{1}\right\}$. Given $\mu \in \mathbf{R}_{+}^{1}$ and $\lambda \in \mathbf{R}_{+}^{1}$, the p.d.f. $f_{2, \mu, \lambda}(x)$ of a gamma-distributed r.v. $T w_{2}(\mu, \lambda)$ is as follows:

$$
f_{2, \mu, \lambda}(x):=\frac{(\lambda / \mu)^{\lambda}}{\Gamma(\lambda)} \cdot x^{\lambda-1} \cdot \exp \{-(\lambda / \mu) \cdot x\}, \text { where } x \geq 0
$$

Every member of the PVF satisfies the following variance-to-mean relation of the power type:

$$
\operatorname{Var}\left(T w_{p}(\mu, \lambda)\right)=\lambda^{-1} \cdot \mathbf{E}\left(T w_{p}(\mu, \lambda)\right)^{p}=\lambda^{-1} \cdot \mathbf{V}_{p}(\mu)=\lambda^{-1} \cdot \mu^{p}
$$


(compare (2.5) and Jørgensen (1997, form. (4.2))). The formula (3.5) justifies employing the name the power-variance family when making a reference to this class, since the variance of r.v. $T w_{p}(\mu, \lambda)$ is proportional to its mean raised to the power $p$.

The following quantity plays a pivotal role for the PVF:

$$
\Phi_{p}\left(=\Phi_{p}(\mu, \lambda)\right):=\lambda \cdot \mu^{2-p}=\mathbf{E}\left(T w_{p}(\mu, \lambda)\right)^{2} / \operatorname{Var}\left(T w_{p}(\mu, \lambda)\right) .
$$

By analogy to Vinogradov (2004a, form. (1.5)), hereinafter it will be referred to as the shape parameter of the law of r.v. $T w_{p}(\mu, \lambda)$.

By analogy to Vinogradov (2004a, form. (1.2)-(1.3)), consider the exponential tilting parameter

$$
\theta_{p}(=\theta(p, \mu, \lambda)):=\frac{1}{p-1} \cdot \lambda \cdot \mu^{1-p}, \text { where } p \in \Delta \backslash\{1\}
$$

Also, in the case where $p \neq 2$, it is often convenient to use the following multiple of $\Phi_{p}$ :

$$
\mathcal{A}_{p}\left(=\mathcal{A}_{p}(\mu, \lambda)\right):=\Phi_{p} /(2-p) .
$$

The consideration of the following quantity is motivated by Vinogradov (2004a, form. (1.4)). For $p \in(1, \infty) \backslash\{2\}$, we introduce the quantity

$$
B_{p, \lambda}:=\frac{(p-1)^{(2-p) /(1-p)}}{2-p} \cdot \lambda^{1 /(p-1)}=\mathcal{A}_{p} \cdot\left(\theta_{p}\right)^{\rho_{p}} .
$$

Since $\forall p \in(-\infty, 0) \cup(2, \infty)$ the law of r.v. $T w_{p}(\mu, \lambda)$ is obtained by an exponential tilting of a particular extreme stable law with index $\alpha_{p}$ given by (2.7), it is absolutely continuous. Its p.d.f., which is hereinafter denoted by $f_{p, \mu, \lambda}(\cdot)$, is given by $(3.14)$.

The positive stable laws with index $\alpha \in(0,1)$ are also included in the PVF as a "border" case corresponding to $\left\{p_{\alpha} \in(2, \infty) ; \mu=\infty\right\}$. Hereinafter, $p_{\alpha}:=(2-\alpha) /(1-\alpha)$. Hence, given $p \in(2, \infty)$ and $y \in \mathbf{R}_{+}^{1}$, it becomes possible to provide the closed-form representation of the p.d.f. $f_{p, \infty, \lambda}(y)$ of a positive stable r.v. $T w_{p}(\infty, \lambda)$ by employing the Wright function. Thus, a combination of Jørgensen (1997, form. (4.19) and comment on p. 141) with (2.2) yields that in the case where $p>2$ and $\lambda \in \mathbf{R}_{+}^{1}$,

$$
f_{p, \infty, \lambda}(y)=y^{-1} \cdot \phi\left(\rho_{p}, 0, B_{p, \lambda} \cdot y^{\rho_{p}}\right), \text { where } y \in \mathbf{R}_{+}^{1} .
$$

In view of the well-known behavior of the Wright function with $\rho \in(-1,0)$ at the origin, the function $f_{p, \infty, \lambda}(\cdot) \in \mathbf{C}\left(\mathbf{R}^{1}\right)$ (compare Zolotarev (1986, form. (2.5.17)-(2.5.18), and Remark 1 on $\mathrm{p}$. 100)).

Similar to the above case, the spectrally negative extreme stable laws with index $\alpha \in(1,2]$ and skewness $\beta=-1$ are included in the PVF corresponding to $\left\{p_{\alpha} \in(-\infty, 0] ; \mu=0\right\}$.

It then follows from Zolotarev (1986, form. (2.4.6)) that $\forall p \in(-\infty, 0], \lambda>0$, and $y \in \mathbf{R}^{1}$, the p.d.f. $f_{p, 0, \lambda}(y)$ of the extreme stable r.v. $T w_{p}(0, \lambda)$ admits the following representation:

$$
f_{p, 0, \lambda}(y) \equiv \begin{cases}y^{-1} \cdot \phi\left(\rho_{3-p}, 0, B_{3-p, \lambda} \cdot y\right) & \text { if } y \in \mathbf{R}^{1} \backslash\{0\} \\ B_{3-p, \lambda} / \Gamma\left(\rho_{3-p}\right) & \text { if } y=0\end{cases}
$$

The functions $x^{-1} \cdot \phi\left(\rho_{p}, 0, B_{p, \lambda} \cdot x^{\rho_{p}}\right)$ and $x^{-1} \cdot \phi\left(\rho_{3-p}, 0, B_{3-p, \lambda} \cdot x\right)$ which emerge in (3.10)-(3.11) can be extended at zero by continuity.

Remark 3.3 Tweedie (1984) did not consider the case where $p<0$ at all. Hence, representation (3.11) for the p.d.f. of a generic member of the subclass of the PVF with $p<0$, which implies 
that the p.d.f. of an extreme stable law with $\alpha \in(1,2)$ is expressed in terms of a specific Wright function in its full domain $\mathbf{R}^{1}$, is new. In this respect, note that Ibragimov and Linnik (1971, Th. 2.3.1) and Zolotarev (1986, Th. 2.4.1) stipulate that in the case where $\alpha \in(0,1)$, the p.d.f. $\mathbf{p}(\cdot)$ of a generic stable law with index $\alpha$ admits the following representation:

$$
\mathbf{p}(y)= \begin{cases}y^{-1} \cdot \epsilon_{1}\left(y^{-\alpha}\right) & \text { if } y>0 \\ y^{-1} \cdot \epsilon_{2}\left((-y)^{-\alpha}\right) & \text { if } y<0\end{cases}
$$

Here $\epsilon_{1}(\cdot)$ and $\epsilon_{2}(\cdot)$ are entire functions. In addition, the assertions quoted above yield that for $\alpha \in(1,2)$, the p.d.f. $\mathbf{p}(\cdot)$ of a generic stable law with index $\alpha$ is an entire function per se. Namely,

$$
\mathbf{p}(y) \equiv \epsilon_{3}(y), \quad \text { where } y \in \mathbf{R}^{1} .
$$

A combination of (3.10)-(3.13) implies that in the extreme stable case for which $\beta= \pm 1$, the functions $\epsilon_{i}(\cdot)$ 's can be identified as some simple transformations of specific Wright functions. We consider that this observation is quite important and has a potential to provide some further insight into various analytical properties of the stable laws. Also, this is in line with the statement made by Zolotarev (1986, p. 167) who wrote that the p.d.f.'s of the univariate stable laws "merit being distinguished as an independent class and accorded "civil rights" in the theory of special functions."

Next, recall that $\forall p \in(-\infty, 0] \cup(2, \infty)$, the p.d.f. $f_{p, \mu, \lambda}(\cdot)$ of $T w_{p}(\mu, \lambda)$ is obtained from the p.d.f.'s $f_{p, 0, \lambda}(\cdot)$ or $f_{p, \infty, \lambda}(\cdot)$, respectively, of certain positive and extreme stable laws, by the exponential tilting. (We refer to Vinogradov (2004a, form. (1.8)-(1.9)) for more detail on this transformation.) A subsequent combination of (3.6)-(3.8) and (3.10)-(3.11) with Vinogradov (2004a, form. (1.8)-(1.9)) implies that $\forall p \in(-\infty, 0] \cup(2, \infty), \forall \lambda \in \mathbf{R}_{+}^{1}$, and $\forall \mu \in \Omega_{p}$,

$$
f_{p, \mu, \lambda}(x) \equiv \begin{cases}x^{-1} \cdot \phi\left(\rho_{p}, 0, B_{p, \lambda} \cdot x^{\rho_{p}}\right) \cdot e^{-\theta_{p} \cdot x-\mathcal{A}_{p}} & \text { if } p>2 \text { and } x \geq 0 \\ x^{-1} \cdot \phi\left(\rho_{3-p}, 0, B_{3-p, \lambda} \cdot x\right) \cdot e^{-\theta_{p} \cdot x-\mathcal{A}_{p}} & \text { if } p \leq 0 \text { and } x \in \mathbf{R}^{1}\end{cases}
$$

It is evident that $\forall p>2, \mu \in(0, \infty], \lambda \in \mathbf{R}_{+}^{1}$, and $\forall x \leq 0$, the p.d.f. $f_{p, \mu, \lambda}(x) \equiv 0$.

Next, in the majority of the works on positive stable distributions, their authors employed the "standardized" positive stable r.v. $\mathcal{X}_{\alpha}$ with index $\alpha \in(0,1)$, whose p.d.f. was denoted by $g(x, \alpha, 1)$ by Zolotarev (1986, p. 13), and whose Laplace transform is equal to $\exp \left\{-t^{\alpha}\right\}$. Note that

$$
\mathcal{X}_{\alpha} \stackrel{\mathrm{d}}{=}\left(-B_{p_{\alpha}, 1}\right)^{-\left(1-p_{\alpha}\right) /\left(2-p_{\alpha}\right)} \cdot T w_{p_{\alpha}}(\infty, 1) .
$$

A subsequent combination of (3.10) with (3.15) implies that $\forall \alpha \in(0,1)$ and $\forall x>0$, the p.d.f.

$$
g(x, \alpha, 1) \equiv x^{-1} \cdot \phi\left(-\alpha, 0,-x^{-\alpha}\right) .
$$

Next, we introduce the following function of the two arguments $\psi \in \mathbf{R}^{1}$ and $\rho \in(-1,0)$ :

$$
\mathcal{W}(\psi, \rho):=\left(\frac{\sin (\rho \cdot \psi)}{\rho \cdot \sin \psi}\right)^{-\rho /(1+\rho)} \cdot \frac{\sin ((1+\rho) \cdot \psi)}{(1+\rho) \cdot \sin \psi}
$$

Remark 3.4 The function $\mathcal{W}(\psi, \rho)$ as well as its variants, were extensively used in stochastic modelling for generating stable random variates (cf., for example, Chambers et al. (1976, form. (2.1)) and Yanushkevichiene (1979, Ch. 3, pp. 85-86 and Tables 3-4 on pp. 92-95)). At the same time, the definition of the analogous function $w(\cdot, \alpha)$ which is employed in Zolotarev $(1986, p .96)$ and Uchaikin and Zolotarev (1999, p. 124) contains an easy-to-fix misprint. 
Now, it follows from Chambers et al. (1976, form. (2.1)) that $\forall \alpha \in(0,1)$ and $x \in \mathbf{R}_{+}^{1}$,

$$
\begin{gathered}
g(x, \alpha, 1) \equiv \frac{\alpha^{-1 /(1-\alpha)}}{2 \pi} \cdot x^{1 /(\alpha-1)} \\
\times \int_{-\pi}^{\pi} \mathcal{W}(\psi,-\alpha) \cdot \exp \left\{-(1-\alpha) \cdot \alpha^{\alpha /(1-\alpha)} \cdot x^{-\alpha /(\alpha-1)} \cdot \mathcal{W}(\psi,-\alpha)\right\} \cdot d \psi
\end{gathered}
$$

The representation (3.18) can be recovered from a more general statement presented in Zolotarev (1986, form. (2.5.10)) or Uchaikin and Zolotarev (1999, form. (4.7.5)).

A combination of (3.16) and (3.18) with some algebra stipulates the following new representation of the Theory of Special Functions: $\forall \rho \in(-1,0)$ and $\forall y>0$,

$$
\begin{gathered}
\phi(\rho, 0,-y) \equiv \frac{(-\rho)^{1 /(1+\rho)}}{2 \pi} \cdot y^{1 /(1+\rho)} \\
\times \int_{-\pi}^{\pi} \mathcal{W}(\psi, \rho) \cdot \exp \left\{-(1+\rho) \cdot(-\rho)^{-\rho /(1+\rho)} \cdot y^{1 /(1+\rho)} \cdot \mathcal{W}(\psi, \rho)\right\} \cdot d \psi
\end{gathered}
$$

Next, consider the case where $p \in(1,2)$. The formula (3.23) stipulates that for these values of $p$, r.v. $T w_{p}(\mu, \lambda)$ has a mixed, compound Poisson-gamma distribution (compare Hougaard (2000, pp. 504-506)). However, prior to giving representation (3.23) we should introduce independent r.v.'s $\left\{\mathcal{H}_{n}, n \geq 1\right\}$ which have a specific common gamma distribution, i.e., $\mathcal{H}_{n} \stackrel{\mathrm{d}}{=} T w_{2}\left(\mu_{\mathcal{H}}, \lambda_{\mathcal{H}}\right)$. Here, $\mu_{\mathcal{H}}$ and $\lambda_{\mathcal{H}}$ are as follows:

$$
\begin{aligned}
\mu_{\mathcal{H}} & :=\rho_{p} / \theta_{p}=(2-p) \cdot \mu^{p-1} / \lambda ; \\
\lambda_{\mathcal{H}} & :=\rho_{p}=(2-p) /(p-1) .
\end{aligned}
$$

In addition, suppose that these gamma-distributed r.v.'s do not depend on the given Poisson r.v. $\mathcal{T} \stackrel{\mathrm{d}}{=} T w_{1}\left(\mu_{\mathcal{T}}, 1\right)$ with mean

$$
\mu_{\mathcal{T}}:=\mathcal{A}_{p}
$$

Then for each $p \in(1,2)$, r.v. $T w_{p}(\mu, \lambda)$ admits the following compound Poisson representation:

$$
T w_{p}(\mu, \lambda) \stackrel{\mathrm{d}}{=} \sum_{k=1}^{\mathcal{T}} \mathcal{H}_{k} .
$$

In the case where $p=3 / 2,(3.20)-(3.21)$ yield that $\mu_{\mathcal{H}}=\sqrt{\mu} /(2 \lambda)$ and $\lambda_{\mathcal{H}}=1$. A combination of this fact with (3.4) implies that $\operatorname{EDM}\left\{T w_{3 / 2}(\mu, \lambda), \mu \in \mathbf{R}_{+}^{1}, \lambda \in \mathbf{R}_{+}^{1}\right\}$ comprises the totality of the class of the Poisson-exponential r.v.'s. See also [VPY2, Example 4.3].

For each $p \in(1,2)$, the law of r.v. $T w_{p}(\mu, \lambda)$ has an absolutely continuous component. It does not have a continuous-singular component, while a combination of (3.22)-(3.23) stipulates that

$$
\mathbf{P}\left\{T w_{p}(\mu, \lambda)=0\right\}=\exp \left\{-\mathcal{A}_{p}\right\}>0 .
$$

It is significant that the Wright function emerges in the formula for the density $f_{p, \mu, \lambda}(\cdot)$ of the absolutely continuous component of r.v. $T w_{p}(\mu, \lambda)$. Thus, it follows by comparing Jørgensen (1997, form. (4.30)-(4.31)) with $(2.2)$ and (3.7)-(3.9) that $\forall p \in(1,2)$ and $\forall y>0$,

$$
f_{p, \mu, \lambda}(y) \equiv y^{-1} \cdot \phi\left(\rho_{p}, 0, B_{p, \lambda} \cdot y^{\rho_{p}}\right) \cdot e^{-\mathcal{A}_{p}-\theta_{p} \cdot y} .
$$


Lemma 3.5 Fix the arbitrary values of $p \in(1,2), \mu \in \mathbf{R}_{+}^{1}$ and $\lambda \in \mathbf{R}_{+}^{1}$. Then

(i) The asymptotic behavior of the lower tail of function $f_{p, \mu, \lambda}(y)$ as $y \downarrow 0$ is as follows:

$$
f_{p, \mu, \lambda}(y):=y^{-1} \cdot e^{-\mathcal{A}_{p}-\theta_{p} \cdot y} \cdot \sum_{k=1}^{\left[1 / \rho_{p}\right] \vee 1} \frac{\left(B_{p, \lambda}\right)^{k}}{k ! \cdot \Gamma\left(\rho_{p} \cdot k\right)} \cdot y^{\rho_{p} \cdot k}+\mathcal{O}\left(y^{\rho_{p} \cdot\left(\left(\left[1 / \rho_{p}\right] \vee 1\right)+1\right)-1}\right) .
$$

(ii) The function $f_{p, \mu, \lambda}(y)$ exhibits the following upper limits at the origin:

$$
f_{p, \mu, \lambda}(0):=\lim _{y \downarrow 0} f_{p, \mu, \lambda}(y)= \begin{cases}0 & \text { if } p \in(1,3 / 2) \\ 4 \lambda^{2} \cdot \exp \left\{-2 \cdot \Phi_{3 / 2}\right\} & \text { if } p=3 / 2 \\ +\infty & \text { if } p \in(3 / 2,2)\end{cases}
$$

Proof of Lemma 3.5. (i) It follows from a combination of (2.3) with (3.25).

(ii) The derivation of (3.27) starting from (3.26) is straightforward.

Now, suppose that $p, \mu$ and $\lambda$ attain their arbitrary fixed admissible values. Fix an arbitrary multiplier $w \in \mathbf{R}_{+}^{1}$. Then Vinogradov (2004a, form. (1.1)) implies that r.v.

$$
\mathcal{S}_{w}:=w \cdot T w_{p}(\mu, \lambda) \stackrel{\mathrm{d}}{=} T w_{p}\left(w \cdot \mu, w^{p-2} \cdot \lambda\right)
$$

A combination of (3.6) with (3.28) stipulates that the shape parameter is multiplication-invariant:

$$
\Phi_{p}\left(\mathcal{S}_{w}\right):=\lambda_{\mathcal{S}_{w}} \cdot \mu_{\mathcal{S}_{w}}^{2-p} \equiv \Phi_{p}
$$

(compare (3.29) with Vinogradov (2004a, form. (2.17))). Also, it follows from (3.28) that under the additional assumption that $\mu \in \Omega_{p} \backslash\{0\}$ is finite, r.v. $T w_{p}(\mu, \lambda)$ satisfies the following relationship:

$$
T w_{p}(\mu, \lambda) \stackrel{\mathrm{d}}{=} \mu \cdot T w_{p}\left(1, \Phi_{p}\right) \stackrel{\mathrm{d}}{=} \lambda^{1 /(p-2)} \cdot T w_{p}\left(\Phi_{p}^{1 /(2-p)}, 1\right)
$$

(compare Vinogradov (2004a, p. 1010)). The rightmost equation in (3.30) is not valid for $p=2$.

The following assertion generalizes and refines Dunn and Smyth (2008, form. (6)).

Proposition 3.6 (i) For each $p \in \Delta, \forall \mu \in \Omega_{p}, \forall \lambda \in \mathbf{R}_{+}^{1}$, for each constant $\mathcal{C} \in \mathbf{R}_{+}^{1}$, and for $y$ belonging to $\mathbf{R}^{1}$, to $[0, \infty)$, and to $\mathbf{R}_{+}^{1}$ in the cases where $p \in(-\infty, 0] \cup(2,+\infty), p=1$, and $p \in(1,2]$, respectively, one ascertains that

$$
f_{p, \mathcal{C} \cdot \mu, \mathcal{C}^{p-2 \cdot \lambda}}(y) \equiv \mathcal{C}^{-1} \cdot f_{p, \mu, \lambda}(y / \mathcal{C})
$$

(ii) In the cases where $p \in(1,3 / 2]$, or $p=2$ and $\lambda \geq 1$, (3.31) remains valid at $y=0$.

Proof of Proposition 3.6. For $p \in \Delta \backslash\{1\}$, both assertions follow from a combination of (3.4), (3.6)-(3.11), (3.14), (3.25), (3.27) with routine calculus. For $p=1$, (3.31) follows from (3.2).

Corollary 3.7 (i) Suppose that $\mu \in \Omega_{p} \backslash\{0 ;+\infty\}$, and all the remaining conditions of Proposition 3.6 are fulfilled. Then

$$
f_{p, \mu, \lambda}(y) \equiv \mu^{-1} \cdot f_{p, 1, \Phi_{p}}(y / \mu) .
$$

(ii) Under the additional assumption that $p \neq 2$,

$$
f_{p, \mu, \lambda}(y) \equiv \mu^{-1} \cdot f_{p, 1, \Phi_{p}}(y / \mu) \equiv \lambda^{1 /(2-p)} \cdot f_{p, \Phi_{p}^{1 /(2-p)}, 1}\left(y \cdot \lambda^{1 /(2-p)}\right) .
$$


Proof of Corollary 3.7. It easily follows by combining (3.6) with (3.31), where one should set $\mathcal{C}$ equal to $\mu$ and to $\lambda^{1 /(p-2)}$ in the proof of parts (i) and (ii), respectively.

In the cases where $\{p>2 ; \mu=+\infty\}$ or $\{p \leq 0, \mu=0\},(3.28)$ implies that

$$
\begin{gathered}
T w_{p}(\infty, \lambda) \stackrel{\mathrm{d}}{=} \lambda^{1 /(p-2)} \cdot T w_{p}(\infty, 1) ; \\
T w_{p}(0, \lambda) \stackrel{\mathrm{d}}{=} \lambda^{1 /(p-2)} \cdot T w_{p}(0,1) .
\end{gathered}
$$

Next, Vinogradov (2004a, Prop. 1.1.iii) implies that $\forall p \in(1,2), \mu \in \mathbf{R}_{+}^{1}$, and $\lambda \in \mathbf{R}_{+}^{1}$,

$$
\zeta_{p, \mu, \lambda}(s):=\log \left(\mathbf{E} \exp \left\{s \cdot T w_{p}(\mu, \lambda)\right\}\right)=\mathcal{A}_{p} \cdot\left\{\left(1-s / \theta_{p}\right)^{-\rho_{p}}-1\right\} \text {, where } s<\theta_{p}
$$

(compare to (4.22)). Also, a combination of Vinogradov (2004a, Th. 2.7.i) with (2.11) implies that for the arbitrary fixed $p \in \Delta \backslash\{1 ; 2\}, \mu \in \Omega_{p}$ and $\lambda \in \mathbf{R}_{+}^{1}$, and for each integer $\ell \geq 1$, the $\ell^{\text {th }}$ cumulant $\mathcal{V}_{p, \mu, \lambda}(\ell)$ of the r.v. $T w_{p}(\mu, \lambda)$ is as follows: $\mathcal{V}_{p, \mu, \lambda}(\ell)=\mathcal{A}_{p} \cdot \theta_{p}^{-\ell} \cdot\left(\rho_{p}\right)_{\ell}$ (compare Withers and Nadarajah (2011, form. (2.5)), which pertains to $p \in(1,2))$.

\section{Refined Saddlepoint-Type Approximations for Tweedie EDM's}

First, given $p \in \Delta$, a combination of (2.10) with Jørgensen (1997, form. (3.44) and (3.48)) and Hougaard (2000, form. (A.21)) suggests the consideration of the following function, which is commonly termed the saddlepoint approximation (for the $\operatorname{EDM}\left\{T w_{p}(\mu, \lambda), \mu \in \Omega_{p}, \lambda \in \mathbf{R}_{+}^{1}\right\}$ ):

$$
\mathcal{F}_{p, \mu, \lambda}(y):=\sqrt{\frac{\lambda \cdot H_{p}^{\prime \prime}(y)}{2 \pi}} \cdot \exp \left\{-\lambda \cdot \mathcal{E}_{p}(y, \mu)\right\}=\sqrt{\frac{\lambda}{2 \pi \cdot y^{p}}} \cdot \exp \left\{-\lambda \cdot \mathcal{E}_{p}(y, \mu)\right\} .
$$

For $p \in \Delta \backslash(2,3)$, consider the following reciprocity transformation of the PVF:

$$
p \rightarrow p^{\prime}:=3-p ; \quad \mu \rightarrow \mu^{\prime}:=1 / \mu ; \quad \lambda \rightarrow \lambda^{\prime}:=\lambda .
$$

A subsequent combination of (2.6) and (4.2) implies that $\forall p \in \Delta \backslash(2,3)$,

$$
\rho_{p^{\prime}}=1 / \rho_{p}
$$

In the case where the reciprocal pair $\left\{p ; p^{\prime}\right\}$ constitutes the two-point set $\{1 ; 2\},(4.3)$ is understood in the generalized sense, as the reciprocal relation between 0 and $\infty$.

Under the assumption that $p \in \Delta \backslash(\{1\} \cup[2,3))$, a combination of (4.2) with (3.7)-(3.8) ascertains that

$$
\mathcal{A}_{p^{\prime}}\left(\mu^{\prime}, \lambda^{\prime}\right)=\theta(p, \mu, \lambda) ; \quad \theta\left(p^{\prime}, \mu^{\prime}, \lambda^{\prime}\right)=\mathcal{A}_{p}(\mu, \lambda) .
$$

Proposition 4.1 Fix a particular value of $y \in \mathbf{R}_{+}^{1}$ and the arbitrary admissible values of $p \in \Delta$, $\mu \in \Omega_{p}, \lambda \in \mathbf{R}_{+}^{1}$.

(i) Consider a certain subfamily of Tweedie laws $\left\{\operatorname{Tw}_{P}(M, \Lambda)\right\}$, where the parameters $P \in \Delta$, $\mathcal{M} \in \Omega_{p}$ and $\Lambda \in \mathbf{R}_{+}^{1}$ vary in such a manner that $P \rightarrow p, \mathcal{M} \rightarrow \mu$, and $\Lambda \rightarrow \lambda$. Then

$$
\mathcal{F}_{P, \mathcal{M}, \Lambda}(y) \rightarrow \mathcal{F}_{p, \mu, \lambda}(y) .
$$

(ii) Under the additional constraint that $p \in \Delta \backslash(\{1\} \cup[2,3))$, the following functions of argument $y$ are identical on $\mathbf{R}_{+}^{1}$ :

$$
\mathcal{F}_{p, \mu, \lambda}(y) \equiv y^{\rho_{p}-1} \cdot \exp \left\{-\theta_{p} \cdot(y-1)+\mathcal{A}_{p} \cdot\left(y^{\rho_{p}}-1\right)\right\} \cdot \mathcal{F}_{3-p, 1 / \mu, \lambda}\left(y^{\rho_{p}}\right) .
$$

(iii) The following functions of argument $y$ are identical on $\mathbf{R}_{+}^{1}$ :

$$
\mathcal{F}_{2, \mu, \lambda}(y) \equiv y^{\rho_{2}-1} \cdot \exp \left\{-\theta_{2} \cdot(y-1)+\lambda \cdot \log y\right\} \cdot \mathcal{F}_{1,1 / \mu, \lambda}\left(y^{\rho_{2}}\right) .
$$


Proof of Proposition 4.1. (i) The validity of (4.5) follows by combining (2.9) and (4.1).

(ii) The proof of (4.6) relies on a combination of (2.9), (3.7)-(3.8) and (4.1)-(4.4).

(iii) The proof of (4.7) involves combining (2.9), (3.7)-(3.8) and (4.1)-(4.3) with some algebra.

Next, we present the following asymptotic series. By analogy to Paris and Kaminski (2001, p. 32), hereinafter we denote the so-called Stirling coefficients by $\left\{\gamma_{k}, k \geq 0\right\}$. An application of formulas (2.1.9)-(2.1.11) therein ascertains that both the gamma function and its reciprocal admit the following (divergent) Poincaré series as $z \rightarrow+\infty$ :

$$
\begin{gathered}
\Gamma(z) \sim \sqrt{2 \pi} \cdot e^{-z} \cdot z^{z-1 / 2} \cdot \sum_{k=0}^{\infty}(-1)^{k} \cdot \gamma_{k} \cdot z^{-k}, \\
\frac{1}{\Gamma(z)} \sim \frac{z^{1 / 2-z} \cdot e^{z}}{\sqrt{2 \pi}} \cdot \sum_{k=0}^{\infty} \gamma_{k} \cdot z^{-k} .
\end{gathered}
$$

By Paris and Kaminski (2001, form. (2.1.12) and Table 2.1), the Stirling coefficients can be determined by recursion. In particular,

$$
\gamma_{0}=1, \gamma_{1}=-\frac{1}{12}, \gamma_{2}=\frac{1}{288}, \gamma_{3}=\frac{139}{51840}, \gamma_{4}=-\frac{571}{2488320}, \gamma_{5}=-\frac{163879}{209018880} .
$$

Next, we present a recursive definition of the Zolotarev polynomials, which are denoted by $\left\{\mathcal{Z}_{k}(\rho), k \in \mathbf{Z}_{+}, \rho \in(-1,0)\right\}$. This definition is essentially due to Zolotarev (1986, p. 95-100) or Uchaikin and Zolotarev (1999, Sec. 4.7). In short, Zolotarev's method relies on the derivation of the asymptotic series for the Laplace integral which emerges in (3.18) with the subsequent application of Zolotarev (1986, Lm. 2.5.1). Also, one needs to use the formulas for the moments of the standard normal distribution.

Definition 4.2 First, we define the auxiliary polynomials $\left\{\widetilde{Q}_{n}(\rho), n \in \mathbf{Z}_{+}\right\}$. To this end, we follow the same lines as Zolotarev (1986, form. (2.5.16)). For each $n \in \mathbf{N}$ and $\rho \in(-1,0)$, define $\widetilde{q}_{n}(\psi, \rho)$ as the scaled coefficient of the Taylor expansion around zero (in even powers of the argument $t$ ) of the following even function of argument $t$, which involves the trigonometric function $\mathcal{W}(\psi, \rho)$ introduced in (3.17):

$$
\exp \left\{\frac{1}{\rho \cdot t^{2}} \cdot\left[\mathcal{W}(\psi \cdot t, \rho)-1+\rho \cdot(\psi \cdot t)^{2} / 2\right]\right\}=1+\sum_{n=1}^{\infty} \widetilde{q}_{n}(\psi, \rho) \cdot t^{2 n} .
$$

(The validity of (4.11) is justified by the fact that the function $\mathcal{W}(\psi, \rho)$ is even in the first argument and $\mathcal{W}^{\prime \prime}(0, \rho)=-\rho$.) Subsequently, we employ Zolotarev (1986, form. (2.5.8)). Specifically, for each $n \in \mathbf{N}$, we define

$$
\widetilde{Q}_{n}(\rho):=\frac{1}{\sqrt{2 \pi}} \cdot \int_{-\infty}^{\infty} \widetilde{q}_{n}(\psi, \rho) \cdot e^{-\psi^{2} / 2} \cdot d \psi .
$$

Also, set $\widetilde{Q}_{0}(\rho) \equiv 1$ and $\mathcal{Z}_{0}(\rho) \equiv 1$ identically. Then the Zolotarev polynomials $\mathcal{Z}_{n}(\rho)$ are defined by the following recursion: $\forall n \in \mathbf{N}$

$$
\mathcal{Z}_{n}(\rho):=-\frac{\rho}{2} \cdot(2 n-1) \cdot \widetilde{Q}_{n-1}(\rho)+\widetilde{Q}_{n}(\rho) .
$$

Remark 4.3 (i) The recursion given in Zolotarev (1986, p. 100) contains a misprint. Namely, his factor $2 n+1$ should be replaced by $2 n-1$.

(ii) It can be shown with some effort that the first five Zolotarev polynomials are as follows:

$$
\mathcal{Z}_{1}(\rho):=-\frac{1}{24} \cdot(\rho+2) \cdot(2 \rho+1)
$$




$$
\begin{gathered}
\mathcal{Z}_{2}(\rho):=\frac{1}{1152} \cdot(\rho+2) \cdot(2 \rho+1) \cdot\left(2 \rho^{2}-19 \rho+2\right), \\
\mathcal{Z}_{3}(\rho):=\frac{1}{414720} \cdot(\rho+2) \cdot(2 \rho+1) \cdot\left(556 \cdot \rho^{4}+1628 \cdot \rho^{3}-9093 \cdot \rho^{2}+1628 \cdot \rho+556\right), \\
\mathcal{Z}_{4}(\rho):=-\frac{1}{39813120} \cdot(\rho+2) \cdot(2 \rho+1) \\
\times\left(4568 \rho^{6}-226668 \rho^{5}-465702 \rho^{4}+2013479 \rho^{3}-465702 \rho^{2}-226668 \rho+4568\right), \\
\mathcal{Z}_{5}(\rho):=-\frac{(\rho+2)(2 \rho+1)}{6688604160}\left(2622064 \rho^{8}+12598624 \rho^{7}-167685080 \rho^{6}-302008904 \rho^{5}\right. \\
\left.+1115235367 \rho^{4}-302008904 \rho^{3}-167685080 \rho^{2}+12598624 \rho+2622064\right) .
\end{gathered}
$$

(iii) All the Zolotarev polynomials contain the factor $(\rho+2) \cdot(2 \rho+1)$ which is manifested in the well-known fact that these polynomials disappear in the normal and the inverse Gaussian cases, for which $p_{\rho}=0$ and 3 , respectively. Also, recall that in the three special cases which pertain to the values of $\rho$ equal to $-2 / 3,-1 / 3$ and 1 , we determine the values of all the Zolotarev polynomials in closed form by using the Pochhammer symbols (see [VPY2, (4.7), (4.15), (4.21]).

(iv) An equivalent and computationally efficient method for the derivation of the Zolotarev polynomials consists in slightly modifying the algorithm presented in Paris and Kaminski (2001, p. 46) or Paris (2010, Appendix A) in a similar setting. However, here we choose to present a slight modification of the method due to Zolotarev, since it is easier to follow. In contrast, Paris' algorithm relies on the techniques developed in the studies of the Wright function and hence, it can be harder to comprehend for experts in Statistics and Probability as compared to Zolotarev's method.

(v) A numerical check, for which Paris' method had been implemented, was run for $1 \leq n \leq 30$. It demonstrates that for all these values of $n$, the polynomial $\mathcal{Z}_{n}(\rho) /((\rho / 2+1) \cdot(2 \rho+1))$ of degree $2 n-2$ has both its leading coefficient and the constant term equal to the respective Stirling coefficient $\gamma_{n}$. See also Remark 4.10 for a related conjecture in the context of the PVF.

Lemma 4.4 (i) For each fixed $\rho \in(-1,0)$ and $\forall n \in \mathbf{N}$, the polynomial $\mathcal{Z}_{n}(\rho)$ as a function of $\rho$ has rational coefficients (which do not depend on $\rho$ ). The common domain of these polynomials can be extended from Zolotarev's range of $\rho \in(-1,0)$ to $\rho \in \mathbf{R}^{1}$.

(ii) For each fixed $\rho \in \mathbf{R}^{1} \backslash\{0\}$ and $\forall n \in \mathbf{N}$,

$$
\mathcal{Z}_{n}(1 / \rho)=\mathcal{Z}_{n}(\rho) / \rho^{2 n}
$$

Proof of Lemma 4.4. See Zolotarev (1986, p. 101, Remark 2).

Corollary 4.5 For each $n \in \mathbf{Z}_{+}, \mathcal{Z}_{n}(\rho)$ is a very special $2 n$-degree polynomial of argument $\rho \in \mathbf{R}^{1}$. Namely, $\mathcal{Z}_{n}(\rho)=c_{2 n}^{(n)} \cdot \rho^{2 n}+c_{2 n-1}^{(n)} \cdot \rho^{2 n-1}+\ldots+c_{1}^{(n)} \cdot \rho+c_{0}^{(n)}$ such that for each integer $0 \leq \ell \leq 2 n$, the coefficients $c_{2 n-\ell}^{(n)}=c_{\ell}^{(n)}$.

Proof of Corollary 4.5. It easily follows by combining Lemma 4.4 with the arguments given in Zolotarev (1986, p. 101, Remark 2) and the fact that his $\alpha=-\rho$.

Lemma 4.6 (i) Fix $\rho \in(0, \infty)$. Then as $z \rightarrow+\infty$,

$$
\begin{aligned}
& \phi(\rho, 0, z) \sim \sqrt{\frac{1}{2 \pi \cdot(1+\rho)}} \cdot(\rho \cdot z)^{1 /(2 \cdot(1+\rho))} \cdot \exp \left\{(1+\rho) \cdot\left(\rho^{-\rho} \cdot z\right)^{1 /(1+\rho)}\right\} \\
& \times \sum_{k=0}^{\infty} \mathcal{Z}_{k}(\rho) \cdot\left((1+\rho) \cdot(\rho \cdot z)^{1 /(1+\rho)}\right)^{-k}
\end{aligned}
$$


(ii) Fix $\rho \in(-1,0)$. Then as $z \rightarrow+\infty$,

$$
\begin{aligned}
& \phi(\rho, 0,-z) \sim \sqrt{\frac{1}{2 \pi \cdot(1+\rho)}} \cdot(-\rho \cdot z)^{1 /(2 \cdot(1+\rho))} \cdot \exp \left\{-(1+\rho) \cdot\left((-\rho)^{-\rho} \cdot z\right)^{1 /(1+\rho)}\right\} \\
& \quad \times \sum_{k=0}^{\infty} \mathcal{Z}_{k}(\rho) \cdot\left((1+\rho) \cdot(-\rho \cdot z)^{1 /(1+\rho)}\right)^{-k} .
\end{aligned}
$$

Proof of Lemma 4.6.ii. It follows with some effort from a combination of (3.16) with Zolotarev (1986, form. (2.5.17)-(2.5.18)).

Recall that the proof of Lemma 4.6.i is deferred to [VPY2, Appendix A].

Remark 4.7 Withers and Nadarajah (2011, p. 16) presented a version of (3.25), with the function $r_{\rho}(y)$ defined by formula (1.2) of that paper being equal to $\phi(\rho, 0, y)$, which is presented by (2.2). Their representation is equivalent to Jørgensen (1997, form. (4.30)-(4.31)). We stress that Withers and Nadarajah (2011) considered the case where $\rho>0$. Apparently, they were unaware of the class of Wright functions. In addition, Withers and Nadarajah (2011, Th. 3.2) contains the asymptotic expansion for their function $r_{\rho}(y)$ as $y \rightarrow+\infty$, which is related to (4.14). Their assertion is of the same character as the result by Wright (1935a, pp. 257-258) who provided the Poincaré series for $\phi(\rho, 0, z)$ as $z \rightarrow+\infty$ with implicitly defined coefficients. The coefficients $\left\{e_{k}, k \geq 0\right\}$ which emerge in Withers and Nadarajah (2011, Th. 3.2) are closely related to the corresponding Zolotarev polynomials. The description of the method of proof of Withers and Nadarajah (2011, Th. 3.2) relies on the techniques of the Bell polynomials. The determination of the coefficients in saddlepoint expansions in terms of Bell polynomials is not new (compare Wojdylo (2006)). It is also similar to Zolotarev (1986, p. 98) who applied this approach in the case of $\rho \in(-1,0)$ (compare (4.15)). It appears that Withers and Nadarajah (2011) were unaware of the monograph by Zolotarev (1986), but their expression for the coefficients is useful. In contrast, the explicit Poincaré series which emerges in (4.14) underscores a relationship between the cases of $\rho>0$ and $\rho \in(-1,0)$.

Theorem 4.8 Fix an arbitrary value of $p \in \Delta$. Then

(i) Fix the arbitrary values of $\mu \in \Omega_{p}$, and $y \in \mathbf{R}_{+}^{1}$. Suppose that $\lambda \rightarrow+\infty$. Then

(i1) In the case where $p=1$ and under the additional constraint that $y \in \lambda^{-1} \cdot \mathbf{N}$, the difference quotient $f_{1, \mu, \lambda}(y)$ admits the following Poincaré series:

$$
f_{1, \mu, \lambda}(y) \sim \mathbf{F}_{1, \mu}(y, \lambda):=\mathcal{F}_{1, \mu, \lambda}(y) \cdot \sum_{k=0}^{\infty} \frac{\gamma_{k}}{(y \cdot \lambda)^{k}} .
$$

(i2) In the case where $p \in \Delta \backslash\{1 ; 2\}$, the function $f_{p, \mu, \lambda}(y)$ admits the following Poincaré series:

$$
f_{p, \mu, \lambda}(y) \sim \mathbf{F}_{p, \mu}(y, \lambda):=\mathcal{F}_{p, \mu, \lambda}(y) \cdot \sum_{k=0}^{\infty} \frac{\mathcal{Z}_{k}\left(\rho_{p}\right) \cdot(p-1)^{2 k}}{\left(y^{2-p} \cdot \lambda\right)^{k}}
$$

(i3) In the case where $p=2$, the p.d.f. $f_{2, \mu, \lambda}(y)$ admits the following Poincaré series:

$$
f_{2, \mu, \lambda}(y) \sim \mathbf{F}_{2, \mu}(y, \lambda):=\mathcal{F}_{2, \mu, \lambda}(y) \cdot \sum_{k=0}^{\infty} \frac{\gamma_{k}}{\lambda^{k}} .
$$

(ii) Fix the arbitrary values of $\mu \in \Omega_{p}$ and $\lambda \in \mathbf{R}_{+}^{1}$. Then

(ii1) If $p=1$ and $y \rightarrow+\infty$ such that $y \in \lambda^{-1} \cdot \mathbf{N}$, (4.16) remains valid.

(ii2) In the case where $p \in(-\infty, 0] \cup(1,2)$ and $y \rightarrow+\infty$, (4.17) is true.

(ii3) In the case where $p \in(2,+\infty)$ and $y \downarrow 0$, (4.17) holds. 
Corollary 4.9 Fix $p \in \Delta$. Suppose that $\mu \in \Omega_{p} \backslash\{0 ;+\infty\}$ and $\lambda \in \mathbf{R}_{+}^{1}$ vary in such a manner that $\Phi_{p}\left(=\lambda \cdot \mu^{2-p}\right) \rightarrow+\infty$, whereas $y$ varies in such a way that there exist constants $K_{1}(p)$ and $K_{2}(p)$, which may depend on $p$, such that $0<K_{1}(p) \leq y / \mu \leq K_{2}(p)<+\infty$. Then

(i) In the case where $p=1$, and under the additional constraint that $y \in \lambda^{-1} \cdot \mathbf{N}$,

$$
f_{1, \mu, \lambda}(y) \sim \mu^{-1} \cdot \mathbf{F}_{1, \mu}\left(y / \mu, \Phi_{1}\right) .
$$

(ii) In the case where $p \in \Delta \backslash\{1\}$,

$$
f_{p, \mu, \lambda}(y) \sim \mu^{-1} \cdot \mathbf{F}_{p, \mu}\left(y / \mu, \Phi_{p}\right) .
$$

Proof of Corollary 4.9. It is obtained by combining Theorem 4.8 with (3.32).

Remark 4.10 We conjecture that for each fixed $k \in \mathbf{Z}_{+}$and as $p \rightarrow 2$,

$$
\mathcal{Z}_{k}\left(\rho_{p}\right) \cdot(p-1)^{2 k} \rightarrow \gamma_{k}
$$

We verified the validity of (4.21) numerically for all non-negative integer $k \leq 30$, since for all such $k$ 's, $\mathcal{Z}_{k}\left(\rho_{2}\right)=\gamma_{k}$. A combination of (4.21) with Proposition 4.1, Lemma 4.4 and Corollary 4.5 would imply that for each fixed $k \in \mathbf{Z}_{+}$, the limits of the $k^{\text {th }}$ term $\mathcal{Z}_{k}\left(\rho_{p}\right) \cdot(p-1)^{2 k} /\left(y^{2-p} \cdot \lambda\right)^{k}$ (of the infinite series which emerges on the right-hand side of (4.17)) as $p \rightarrow 2$ and as $p \downarrow 1$ coincide with the corresponding $k^{\text {th }}$ terms of the infinite series which emerge on the right-hand sides of (4.18) and (4.16), respectively. For a fixed $k \in \mathbf{Z}_{+}$, this would imply the "term-wise" continuity of the family of the $k^{\text {th }}$ terms of the corresponding Poincaré series with respect to the parameter $p$.

Remark 4.11 Dunn and Smyth (2005, 2008) considered two different methods for approximating $f_{p, \mu, \lambda}(y)$. Although they addressed the case of $p>2$ as well, here we concentrate on the case where $p \in(1,2)$. Their papers provide the first rigorous studies on the accuracy of such approximations, which is consistent with a statement made in Dunn and Smyth (2008, p. 74). The first method proposed in Dunn and Smyth (2005) allows an equivalent reformulation in terms of a truncation of the infinite series which emerges in (2.2). This is similar to (2.3) and (3.26). Also, Dunn and Smyth (2008, p. 74) stated that the "series" method used in their 2005 paper underperforms for large values of $y$. Their second approach pursued in Dunn and Smyth (2008), which they termed the "inversion" method, relies on the inversion formula for the p.d.f. of $T w_{p}(\mu, \lambda)$ conditioned to be positive. The justification of such an approach involves the use of the ch.f. of this r.v., which is denoted by $\left(T w_{p}(\mu, \lambda) \mid T w_{p}(\mu, \lambda)>0\right)$. An expression for the ch.f. of the latter r.v. is obtained by combining (3.24) with the following representation for the ch.f. of $T w_{p}(\mu, \lambda)$ :

$$
\mathbf{E} \exp \left\{i \cdot s \cdot T w_{p}(\mu, \lambda)\right\}=\exp \left\{\mathcal{A}_{p} \cdot\left(\left(1-i \cdot s / \theta_{p}\right)^{-\rho_{p}}-1\right)\right\}
$$

(compare (3.36)). A combination of (3.24)-(3.25) implies that the r.v. $\left(T w_{p}(\mu, \lambda) \mid T w_{p}(\mu, \lambda)>0\right)$ is absolutely continuous with its p.d.f. being a multiple of $f_{p, \mu, \lambda}(y)$. In view of (4.22), there is a pitfall in an argument by Dunn and Smyth (2008), since for $p \in(3 / 2,2)$, the ch.f. of $\left(T w_{p}(\mu, \lambda) \mid T w_{p}(\mu, \lambda)>0\right)$ is not a member of $\mathbf{L}^{1}$. This is consistent with the fact that by (3.27), $f_{p, \mu, \lambda}(y)$ is unbounded in the rightmost neighborhood of zero for the same range of values of p. In turn, this implies that in the case where $p \in(3 / 2,2)$, one cannot obtain the validity of Dunn and Smyth (2008, form. (10)) automatically. We conjecture that it should be possible to fill in this gap by employing a modification of the machinery developed by Vinogradov (2007b, p. 407). 


\section{References}

[1] Braaksma, B.L.J. (1963). Asymptotic expansions and analytic continuations for a class of Barnes integrals. Compos. Math. 15:239-341.

[2] Chambers, J.M., Mallows, C.L., Stuck, B.W. (1976). A method for simulating stable random variables. JASA 71:340-344.

[3] Dunn, P.K., Smyth, G.K. (2005). Series evaluation of Tweedie exponential dispersion model densities. Stat. Comput. 15:267-280.

[4] Dunn, P.K., Smyth, G.K. (2008). Evaluation of Tweedie exponential dispersion model densities by Fourier inversion. Stat. Comput. 18:73-86.

[5] Gorenflo, R., Luchko, Yu., Mainardi, F. (1999). Analytical properties and applications of the Wright function. Fract. Calc. Appl. Anal. 2:383-414.

[6] Hochberg, K.J., Vinogradov, V. (2009). Structural, continuity and asymptotic properties of a branching particle system. Lith. Math. J. 49:241-270.

[7] Hougaard, P. (2000). Analysis of Multivariate Survival Data. New York: Springer.

[8] Ibragimov, I.A., Linnik, Yu.V. (1971). Independent and Stationary Sequences of Random Variables. Groningen: Wolters-Noordhoff.

[9] Ioffe, A.D., Tihomirov, V.M. (1979). Theory of Extremal Problems. New York: North-Holland.

[10] Jørgensen, B. (1997). The Theory of Dispersion Models. London: Chapman \& Hall.

[11] Jørgensen, B., Martínez, J.R., Vinogradov, V. (2009). Domains of attraction to Tweedie distributions. Lith. Math. J. 49:399-425.

[12] Olver, F. W. J. (1997). Asymptotics and Special Functions. New York: Academic Press.

[13] Paris, R.B. (2010). Exponentially small expansions in the asymptotics of the Wright function. J. Comput. Appl. Math. 234:488-504.

[14] Paris, R.B. (2011a). Hadamard Expansions and Hyperasymptotic Evaluation: An Extension of the Method of Steepest Descents. Cambridge: Cambridge University Press.

[15] Paris, R.B. (2011b). Exponential smoothing of the Wright function. Technical Report MS 11:01. Dundee: University of Abertay.

[16] Paris, R.B., Kaminski, D. (2001). Asymptotics and Mellin-Barnes Integrals. Cambridge: Cambridge University Press.

[17] Poincaré, H. (1886). Sur les intégrales irrégulières des équations linéaires.Acta Math. 8:295-344.

[18] Tweedie, M.C.K. (1984). An index which distinguishes between some important exponential families. In: Ghosh, J.K., Roy, J., eds. Statistics: Applications and New Directions. Proceedings of the Indian Statistical Institute Golden Jubilee International Conference, Calcutta: Indian Statistical Institute, pp. 579-604.

[19] Uchaikin, V.V., Zolotarev, V.M. (1999). Chance and Stability. Utrecht: VSP.

[20] Vinogradov, V. (2004a). On the power-variance family of probability distributions. Commun. Statist. Theor. Meth. 33:1007-1029 (errata p. 2573).

[21] Vinogradov, V. (2007a). On infinitely divisible exponential dispersion model related to Poisson-exponential distribution. Commun. Statist. Theor. Meth. 36:253-263.

[22] Vinogradov, V. (2007b). Local theorems related to Lévy-type branching mechanism. Commun. Stoch. Anal. $1: 393-413$. 
[23] Vinogradov, V., Paris, R.B., Yanushkevichiene, O.L. (2012b). New properties and representations for members of the power-variance family. II. Lith. Math. J. (in press).

[24] Withers, C.S., Nadarajah, S. (2011). On the compound Poisson-gamma distribution. Kybernetika 47:15-37.

[25] Wojdylo, J. (2006). On the coefficients that arise from Laplace's method. J. Comput. Appl. Math. 196:241-266.

[26] Wright, E. M. (1935a). The asymptotic expansion of the generalized Bessel function. Proc. London Math. Soc. (Ser. II) $38: 257-250$.

[27] Yanushkevichiene, O.L. (1979). The Parameter Estimators for the Distributions of Stable Laws. Ph.D. Thesis. Vilnius: Vilnius University (in Russian).

[28] Zolotarev, V.M. (1986). One-dimensional Stable Distributions. Providence, RI: AMS. 